Proceedings

\title{
Fully Printed Wearable Electrode Textile for Electrotherapy Application †
}

\author{
Meijing Liu *, Monika Glanc-Gostkiewicz, Steve Beeby and Kai Yang
}

Citation: Liu, M.; Glanc-Gostkiewicz, M.; Beeby, S.; Yang, K. Fully Printed Wearable Electrode Textile for Electrotherapy Application. Proceedings 2021, 68, 12. https://doi.org/10.3390/proceedings2021068012

Published: 18 January 2021

Publisher's Note: MDPI stays neutral with regard to jurisdictional claims in published maps and institutional affiliations.

Copyright: $(2021$ by the authors. Licensee MDPI, Basel, Switzerland. This article is an open access article distributed under the terms and conditions of the Creative Commons Attribution (CC BY) license (http://creativecommons.org/licenses/by/4.0/).

\author{
Smart Electronic Materials and Systems Research Group, School of Electronics and Computer Science, \\ University of Southampton, Southampton SO17 1BJ, UK; mgg1y14@soton.ac.uk (M.G.-G.); \\ spb@ecs.soton.ac.uk (S.B.); ky2e09@soton.ac.uk (K.Y.) \\ * Correspondence: ml5y17@soton.ac.uk \\ + Presented at the International Conference on the Challenges, Opportunities, Innovations and Applications \\ in Electronic Textiles (E-Textiles 2020), Virtual venue, UK, 4 November 2020.
}

\begin{abstract}
Electrotherapy is a common therapeutic treatment used in pain relief. This paper presents the materials and fabrication methods used to manufacture an electrode textile for electrotherapy application. The Young's modulus of the electrode is $0.22 \mathrm{MPa}$. The electrode textile consists of conductive tracks sandwiched between an interface layer and an encapsulation layer, and an electrode layer printed directly on top of the conductive grid patterns. The interface, conductive silver, and encapsulation layers were directly printed on fabric using screen printing. The electrode layer was printed using stencil printing. The electrode textile can survive 10,000 bending cycles around a cylinder with a diameter of $30 \mathrm{~mm}$ and 20 washes in a commercial washing machine.
\end{abstract}

Keywords: electrotherapy; E-textile; screen printing; stencil printing; electrode; durability

\section{Introduction}

Electrotherapy is a common therapeutic treatment which applies 0-100 milliampere current through electrodes placed on the skin to stimulate the nerve and interfere with the transmission of pain signals. It can be used in pain relief for knee osteoarthritis which will affect 8.3 million people in the UK by 2035 [1].

An electrotherapy device consists of two parts including an electronic control unit to control the current parameters and electrodes to deliver the current. Traditional electrotherapy devices use gel electrodes which are not compatible with textiles [2]. It is recommended gel electrodes are kept in sealed bags after use to maintain the moisture level. The standard lifetime for the gel electrodes is only 2-4 weeks due to the drying out of the electrode and the electrode contamination.

In previous work, a novel dry electrode (Fabink E-0002) was developed by the University of Southampton research group [3]. The soft and tacky (but not sticky) novel electrode combines the advantages of the dry electrode (e.g., easy to use, easy to maintain) and gel electrode (e.g., good skin contact, no hot spots) [4]. This work presents the fabrication process of an electrode textile, and its mechanical property and durability during bending and washing.

\section{Materials}

The polyester/cotton (A1656, plain weaving, $165 \mu \mathrm{m}$ thick) used in this study was purchased from Whaleys Bradford Ltd. (Bradford, UK). The functional pastes for screen printing listed in Table 1 were supplied by Smart Fabric Inks Ltd. (Southampton, UK). 
Table 1. Pastes and their functionality.

\begin{tabular}{cc}
\hline Pastes & Functionality \\
\hline Fabink UV-IF-1004 & Standard interface to create smooth surface on various fabrics \\
Fabink UV-IF-1039 & Waterproof interface and encapsulation suitable for various fabrics \\
Fabink TC-C4007 & Silver ink for printing flexible conductive layer on top of the interface layer \\
Fabink E-0002 & Carbon rubber paste for soft and tacky (but not sticky) electrode \\
Binder material of the novel electrode paste & Rubber to protect silver tracks and improve the electrode-fabric adhesion \\
\hline
\end{tabular}

\section{Methods}

\subsection{Tensile Test}

The equipment used for the tensile test was the Tinius Olsen H25KS tensometer. As shown in Figure 1, the electrode sample was clamped in a vertical direction using two grips. The tensile force on the grips was recorded during the test. The tensile stress was calculated by the software as a part of the testing system.

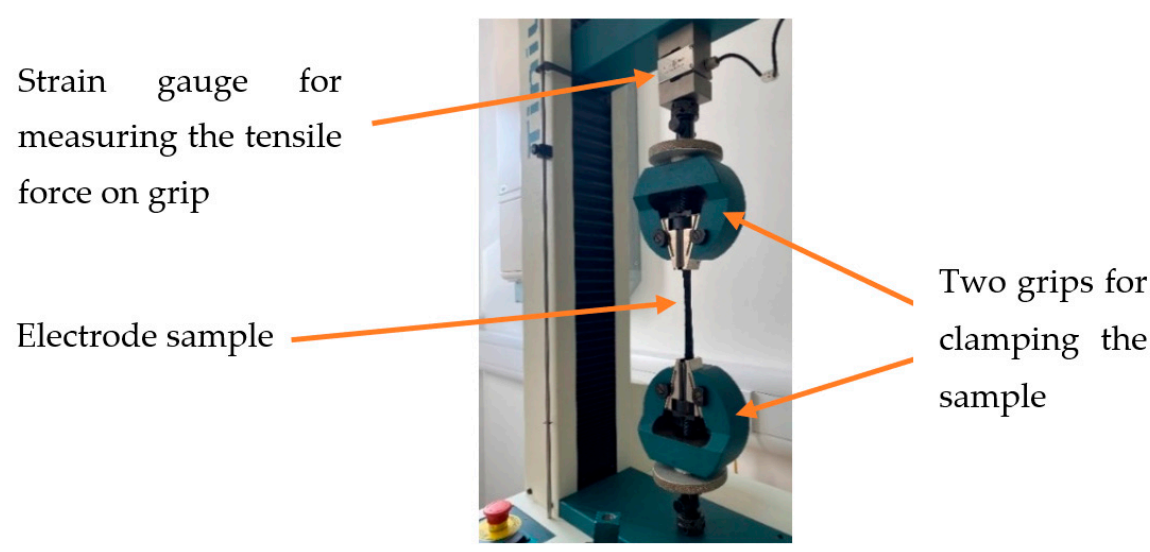

Figure 1. Tinius Olsen H25KS tensometer. An electrode sample is being measured.

\subsection{Printing Method}

A DEK248 semi-automatic screen printer shown in Figure 2a was used to print the interface, conductive silver, and encapsulation layers. Screens were supplied by MCI Precision Screens Ltd. (Melbourn, UK). Figure $2 \mathrm{~b}$ shows the schematic diagram of stencil printing which was used to fabricate the electrode layer. The paste was placed in a $6 \mathrm{~cm} \times$ $6 \mathrm{~cm} \times 2 \mathrm{~mm}$ aluminum frame and casted using a squeegee to form a smooth surface.

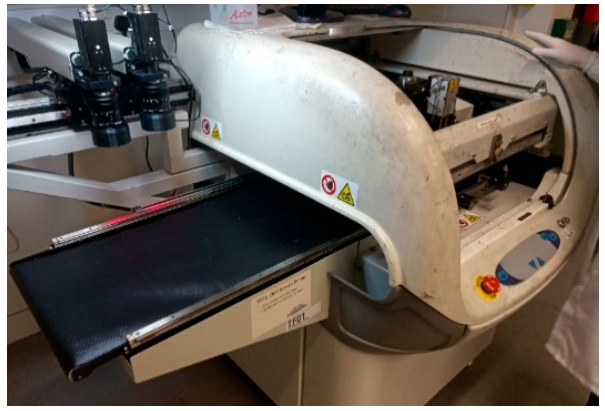

(a)

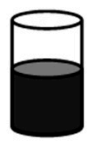

Paste

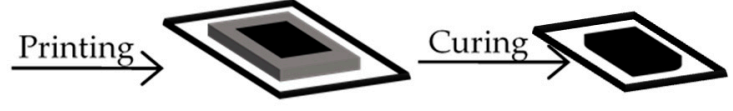

Carbon paste in frame

Electrode

(b)

Figure 2. (a) The DEK 248 screen printer; (b) the schematic diagram for stencil printing. 


\section{Results and Discussion}

\subsection{Tensile Test}

Figure 3 shows the tensile stress-strain curve of the electrode. The stress increases with strain from 0 to until the sample was broken. Young's modulus is calculated as the slope of the linear fitting line, where the value is $0.22 \mathrm{MPa}$. The elongation is $355 \%$.

For reference, the Young's modulus of the skin is between $0.42 \mathrm{MPa}$ and $0.85 \mathrm{MPa}$ [5]. The electrode is softer than skin and provides good conformability without constraining body movement or causing discomfort during movement.

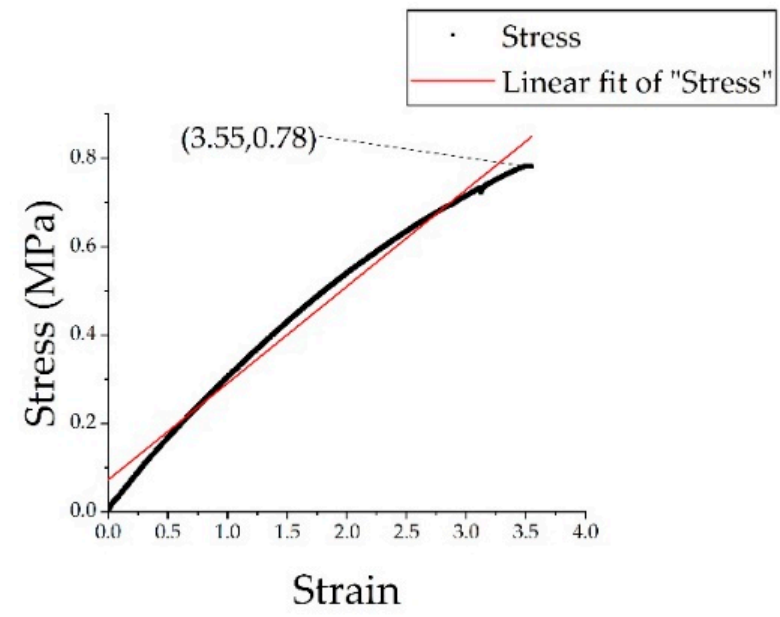

Figure 3. The tensile stress-strain curve of novel dry electrode.

\subsection{Electrode Textile}

\subsubsection{Fabrication Process}

An electrode textile was fabricated by printing the five layers in the following order:

(1) Interface layer to create a smooth and flexible surface on which the silver paste can be printed (Figure 4a). The interface layer was formed using two prints of Fabink IFUV-1039 followed one print of Fabink IF-UV-1004. The Fabink IF-UV-1039 provides a waterproof layer and the Fabink UV-IF-1004 creates a smooth surface with good adhesion to the silver layer. Ultraviolet (UV) curing was applied after each print by exposing the sample to a UV cabinet supplied by UV Light Technology Ltd. (Birmingham, UK). The UV curing time was $60 \mathrm{~s}$ for Fabink IF-UV-1039 and $30 \mathrm{~s}$ for Fabink IF-UV1004.

(2) Conductive silver layer to create the conductive tracks and conductive grid patterns shown in Figure $4 \mathrm{~b}$. The conductive layer was formed using one print of Fabink TCC4007. The paste was cured in a box oven at $130{ }^{\circ} \mathrm{C}$ for $25 \mathrm{~min}$.

(3) Encapsulation layer printing to create a protective layer for the conductive tracks (Figure 4c). The encapsulation layer was formed using one print of Fabink IF-UV1004 followed by two prints of Fabink IF-UV-1039. Curing time was the same as those used in the interface layer.

(4) Carbon rubber layer to provide an interface between the conductive pads and the skin (Figure 4d). The electrode layer was directly printed on top of conductive grid patterns using stencil printing. The electrode paste was cured in a box oven at $80{ }^{\circ} \mathrm{C}$ for $30 \mathrm{~min}$.

(5) Rubber layer printing around the electrode edges and silver tracks to provide electrode adhesion to the fabric and protect the conductive tracks (Figure 4e). The rubber layer was printed by hand with a syringe and cured in a box oven at $80^{\circ} \mathrm{C}$ for $20 \mathrm{~min}$. 


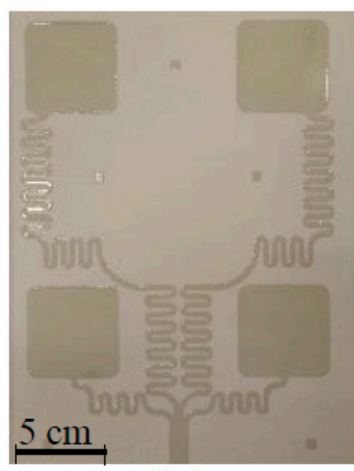

(a)

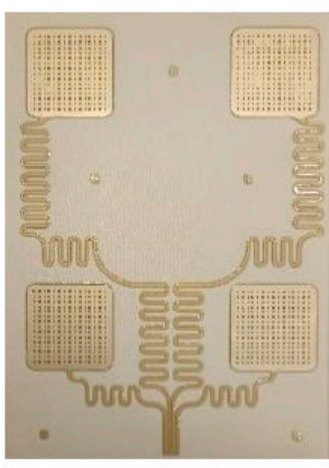

(b)

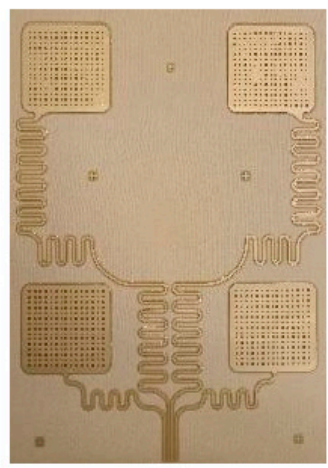

(c)

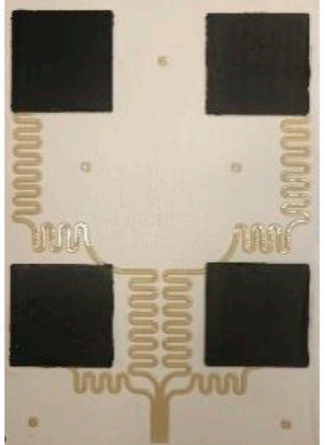

(d)

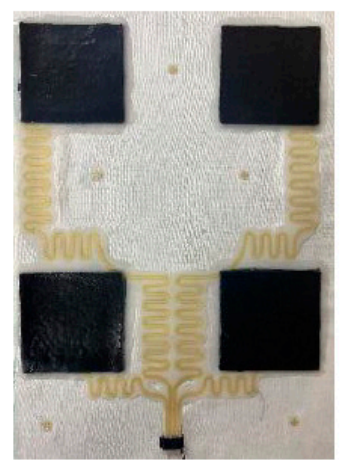

(e)

Figure 4. Electrode textile printing process: (a) interface layer; (b) conductive silver layer; (c) encapsulation layer; (d) carbon rubber layer; (e) rubber layer.

\subsubsection{Durability Test}

The electrode textile was tested on a bending machine (Figure 5a). The diameter of the cylinder was $30 \mathrm{~mm}$. The resistance change during the bending test is shown in Figure $5 \mathrm{~b}$. The initial resistance was $223.4 \pm 51 \Omega$. After 5,000 bending cycles, the samples resistance was $393.7 \pm 86 \Omega$. After 10,000 bending cycles, the samples resistance values varied from $500 \Omega$ to $1,500 \Omega$.

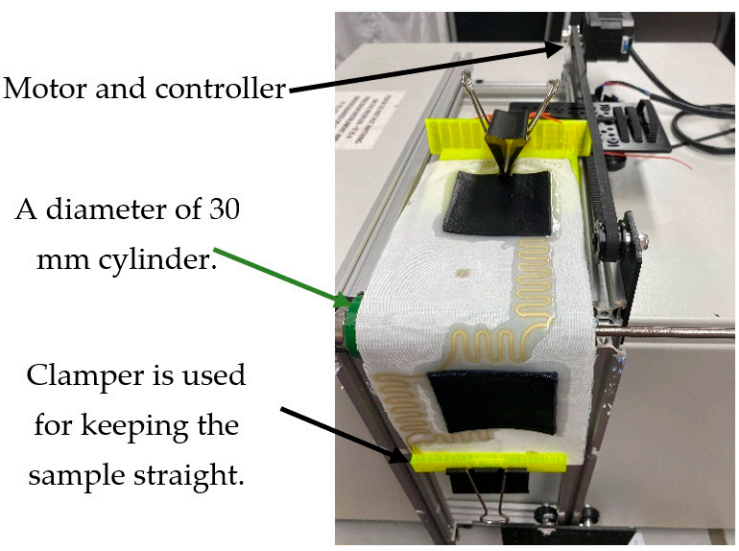

(a)

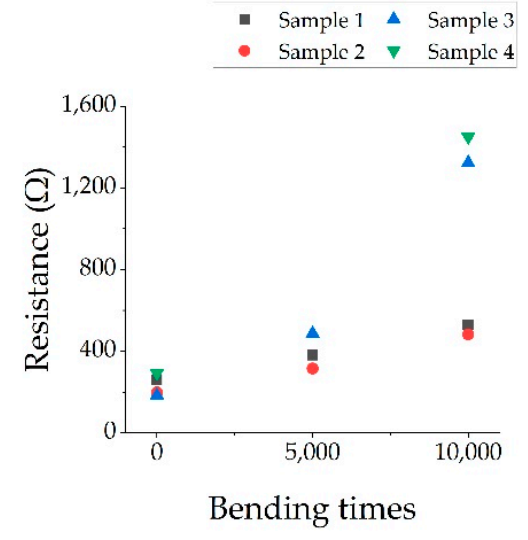

(b)

Figure 5. (a) Bending test set-up; (b) the change of electrode resistance during bending test.

The electrode textile was placed in a wash basket and washed with a lab coat in a commercial washing machine WME7247 supplied by Beko plc (UK). The washing time was $49 \mathrm{~min}$ with a spin speed at 1,000 round per minute and water temperature at $30^{\circ} \mathrm{C}$. The initial resistance of electrode textile samples was $248.3 \pm 46 \Omega$. After 20 washes, the sample's resistance was $342.9 \pm 52 \Omega$. The resistance of the sample increased around 100 $\Omega$ after 20 washing cycles. The increasing resistance will increase the power consumption in electrotherapy application. However, functionality was still achieved after 20 washes.

\section{Conclusions and Future Work}

This paper presents the fabrication process of an electrode textile used in electrotherapy for pain relief. The Young's modulus of the dry electrode was $0.22 \mathrm{MPa}$ which is softer than human skin providing good conformability and comfort in wearable applications. The electrode could be stretched to $355 \%$ before it broke. The electrode textile survived 10,000 bending cycles at a diameter of $30 \mathrm{~mm}$ cylinder and 20 washes in a commercial 
washing machine. The electrode textile will be tested in wearable electrotherapy applications to reduce knee joint pain.

Author Contributions: M.L. carried out the experiments and wrote the manuscript draft. M.G.-G. helped with the electrode textile fabrication. S.B. co-supervised the research. K.Y. provided supervision for the overall research work and edited the manuscript. All authors have read and agreed to the published version of the manuscript.

Funding: This research was funded by EPSRC, grant number EP/S001654/1.

Institutional Review Board Statement: Not applicable.

Informed Consent Statement: Not applicable.

Data Availability Statement: The data presented in this study are openly available in FigShare at http://doi.org/10.6084/m9.figshare.13547117.

Conflicts of Interest: K.Y. and S.B. are directors of Smart Fabric Inks.

\section{References}

1. Osteoarthritis in General Practice-Data and Perspectives-Arthritis Research UK. 2013. Available online: https://www.bl.uk/collection-items/osteoarthritis-in-general-practice-data-and-perspectives (accessed on 5 November 2020).

2. Liu, M.; Beeby, S.; Yang, K. Electrode for Wearable Electrotherapy. Proceedings 2019, 32, 5, doi:10.3390/proceedings2019032005.

3. Yang, K.; Meadmore, K.; Freeman, C.; Grabham, N.; Hughes, A.M.; Wei, Y.; Torah, R.; Glanc-Gostkiewicz, M.; Beeby, S.; Tudor, J. Development of User-Friendly Wearable Electronic Textiles for Healthcare Applications. Sensors 2018, 18, 2410, doi:10.3390/s18082410.

4. Liu, M.; Ward, T.; Young, D.; Matos, H.; Wei, Y.; Adams, J.; Yang, K. Electronic textiles based wearable electrotherapy for pain relief. Sens. Actuators A Phys. 2019, 111701, doi:10.1016/j.sna.2019.111701.

5. Pawlaczyk, M.; Lelonkiewicz, M.; Wieczorowski, M. Age-dependent biomechanical properties of the skin. Adv. Dermatol. Allergol. 2013, 5, 302-306, doi:10.5114/pdia.2013.38359. 